\title{
Enhancements for Monte-Carlo Tree Search in Ms Pac-Man
}

\author{
Tom Pepels
}

\author{
Mark H.M. Winands
}

\begin{abstract}
In this paper enhancements for the Monte-Carlo Tree Search (MCTS) framework are investigated to play Ms Pac-Man. MCTS is used to find an optimal path for an agent at each turn, determining the move to make based on randomised simulations. Ms Pac-Man is a real-time arcade game, in which the protagonist has several independent goals but no conclusive terminal state. Unlike games such as Chess or Go there is no state in which the player wins the game. Furthermore, the Pac-Man agent has to compete with a range of different ghost agents, hence limited assumptions can be made about the opponent's behaviour. In order to expand the capabilities of existing MCTS agents, five enhancements are discussed: 1) a variable depth tree, 2) playout strategies for the ghost-team and Pac-Man, 3) including long-term goals in scoring, 4) endgame tactics, and 5) a Last-Good-Reply policy for memorising rewarding moves during playouts. An average performance gain of 40,962 points, compared to the average score of the top scoring Pac-Man agent during the CIG'11, is achieved by employing these methods.
\end{abstract}

\section{INTRODUCTION}

Ms Pac-Man is a real-time arcade game based on the popular Pac-Man game. The player controls the main character named Ms Pac-Man (henceforth named Pac-Man) through a maze, eating pills and avoiding the ghosts chasing her. The maze contains four so-called power-pills that allow the player to eat the ghosts to obtain a higher score. When all pills in a maze are eaten, the game progresses to the next level. Ms Pac-Man inherited its game-mechanics from the original PacMan. Moreover, it introduced four different mazes, and more important, unpredictable ghost behaviour. This last feature makes Ms Pac-Man an interesting subject for AI research. The game rules are straightforward, however complex planning and foresight are required for a player to achieve high scores.

Two competitions are held for autonomous Ms Pac-Man agents. In the first, Ms Pac-Man Competition (screen-capture version) [1], the original version of the game is played using an emulator. Agents interpret a capture of the screen to determine the game's state. Each turn moves are passed to the emulator running the game. The second, Ms Pac-Man vs Ghosts Competition [2] offers a complete implementation of the game, in which the game state is fully available. Furthermore, Pac-Man agents compete with a variety of ghostteam agents also entered in the competition.

Although most Pac-Man agents entering the competitions are rule-based, research has been performed on using techniques such as genetic programming [3], neural networks [4]

Tom Pepels is a student member of the Games and AI Group, Department of Knowledge Engineering, Faculty of Humanities. Email: thj.pepels@student.maastrichtuniversity.nl; Mark Winands is a member of the Games and AI Group, Department of Knowledge Engineering, Faculty of Humanities and Sciences, Maastricht University, Maastricht, The Netherlands. Email: m.winands@maastrichtuniversity.nl and search trees [5]. Owing to the successful application of Monte-Carlo Tree Search (MCTS) in other games [6], interest in developing MCTS agents for Ms Pac-Man has grown. Samothrakis et al. [7] developed an MCTS agent using a $\operatorname{Max}^{n}$ tree with scoring for both Pac-Man and the ghosts. Furthermore, a target location is set as a long-term goal for Pac-Man, MCTS computes the optimal route to the target in order to determine the next move. Other MCTS-based agents were researched for achieving specific goals in Ms PacMan, such as ghost avoidance [8] and endgame situations [9] demonstrating the possibilities of MCTS for Pac-Man agents. In 2011 the first MCTS agent won the Ms Pac-Man screencapture competition [1]. Until then rule-based agents lead the competitions. The victorious MCTS agent, NOZOMU [10], was designed to avoid so-called 'pincer moves', in which every escape path for Pac-Man is blocked. The approach was successful in beating the leading rule-based agent ICE PAMBUSH [11] with a high score of 36,280. In the same year, the first MCTS based agents entered the Ms Pac-Man vs Ghosts Competition. Both a ghost team agent $[12,13]$ and a Pac-Man agent [14] based on a combination of knowledge rules and MCTS search entered the competition. Moreover, the ghost team agent NQKIEN [12] won the CEC11 [15] edition of the competition with an average score of 11,407.

A Pac-Man agent for the Ms Pac-Man vs Ghosts Competition is developed for this paper, with the goal of a generally strong playing agent. To enhance the MCTS framework for Pac-Man five enhancements are introduced: 1) a variable depth tree, 2) playout strategies for the ghost-team and Pac-Man, 3) including long-term goals in scoring, 4) endgame tactics, and 5) a Last-Good-Reply policy [16] for memorising rewarding moves during playouts.

The paper is structured as follows. First, the Ms PacMan framework is introduced. Next, MCTS and the UCT selection policy are described, and enhancements to the MCTS framework discussed in detail. Finally, experimental results will be given and a conclusion drawn.

\section{MS PAC-MAN}

The basic rules of Ms Pac-Man are based on the classic arcade game. Pac-Man initially has three lives, which she loses through contact with a non-edible ghost. In this case, the location of the ghosts and Pac-Man are reset to their initial configuration. The game environment consists of four different mazes, each is played once per four levels. The game progresses every time unit, allowing Pac-Man to make a move, however, ghosts are only allowed to make a move at a junction. On a path between junctions ghosts can only travel forward. When a power-pill is eaten by Pac-Man the ghosts become 


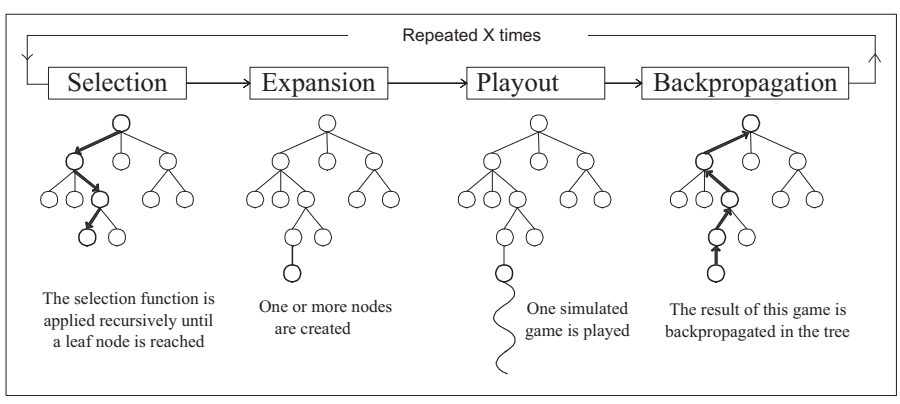

Fig. 1. Strategic steps of Monte-Carlo Tree Search [17]

edible, their movement speed decreases and they are instantly forced to reverse their direction. When Pac-Man reaches a score of 10,000 by eating pills and ghosts, she gains a life. Both the ghosts and Pac-Man have one in-game time unit of $40 \mathrm{~ms}$. to compute a move at each turn. If no move is returned, a randomly selected move is executed. Furthermore, at each turn with a small probability of 0.0015 each ghost is immediately forced to reverse its direction. Each maze is played for 3,000 time units, after which the game progresses to the next level. Remaining pills in the maze are then added to Pac-Man's score as a reward for surviving the maze. The time that ghosts remain edible is decreased when the game advances to the next level. The game ends if either the $16^{\text {th }}$ level is cleared or if Pac-Man has no lives remaining.

\section{Monte-Carlo Tree Search}

Monte-Carlo Tree Search (MCTS) is a best-first search method based on random sampling by Monte-Carlo simulations of the state space for a certain domain $[18,19]$. In gameplay this means that decisions are made based on the results of randomly simulated playouts. MCTS has shown promising results when applied to various turn-based games such as Go [20] and Hex [21]. However, the method can be applied to other problems for which the state space can be represented as a tree. A particular challenge for agents playing real-time games is that they are usually characterised by uncertainty, a large state space and open-endedness. However, MCTS copes well when limited time is available between moves and is possible to encapsulate uncertainty in its randomised playouts [6]. The basic version of MCTS consists of four steps, which are performed iteratively until a computational threshold is reached, i.e. a set number of iterations, an upper limit on memory usage or a time constraint. The four steps (Figure 1) at each iteration are [17]:

- Selection. Starting at the root node, children are selected recursively according to a selection policy. When a leaf node is reached that does not represent a terminal state it is selected for expansion.

- Expansion. All children are added to the selected leaf node given available moves.

- Playout. A simulated playout is run, starting from the state of the added node. Moves are performed randomly or according to a heuristic strategy until a terminal state is reached.

- Backpropagation. The result of the simulated playout is propagated immediately from the selected node back up to the root node. Statistics are updated along the tree for each node selected during the selection phase and visit counts are increased.

Because results are immediately backpropagated, MCTS can be terminated anytime to determine the decision to be made. Moreover, no static heuristic evaluation is required when simulations are performed. However, it is often beneficial to add domain knowledge for choosing moves made during the playout.

\section{Monte-Carlo Tree Search for Ms PaC-Man}

This section discusses the enhancements to the MCTS framework for the Pac-Man agent. The agent builds upon the methods proposed in [10] as well as [7]. The structure of the search tree is defined and the following subsections cover the individual enhancements.

\section{A. Search Tree and Variable Depth}

The game's environment is consists of four different mazes. These can directly be represented as a graph where the junctions are nodes, and paths between junctions are edges. Pac-Man has the option to make a decision at any location in the graph. At a node she has a choice between more than two available directions, while on an edge she can choose to maintain her course or reverse. An example of such a graph is depicted in Figure 2. The associated search tree is shown in Figure 3.

Decisions in the tree are the moves made at nodes, i.e. junctions in the maze. Traversing the tree means that PacMan moves along an edge until a node is reached. At this point either the tree ends and playout starts, or a new edge is chosen based on a child of the current node.

Within the tree, reverse moves, i.e. moves that lead back to a parent, are not considered past the first ply. When a node $n_{p}$, representing junction $j_{p}$ is expanded, each child $n_{i}$ represents a move that leads to a different junction $j_{i}$ in the

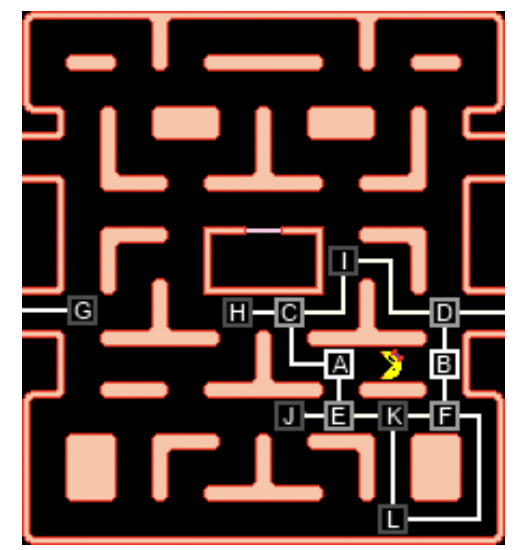

Fig. 2. Graph representation of a game state. 


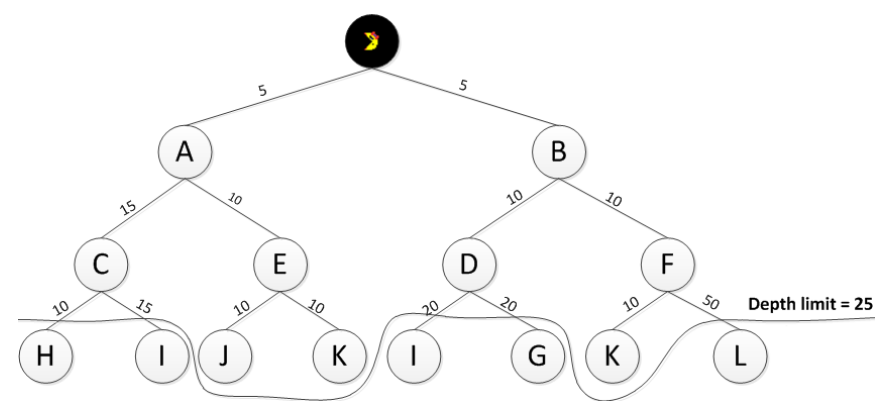

Fig. 3. Example tree with variable tree-depth of 25. Based on the game state in Figure 2.

maze, excluding junction $j_{p}$. Reverse moves are not considered deeper in the tree to ensure a larger difference in rewards between available moves. If reverse moves are expanded on every ply, more playouts per move are required in order to have conclusive differences in rewards. Considering that: 1) in total, more nodes are expanded, and 2) the tree contains duplicate paths with similar or equal rewards.

Nodes store three reward values both averaged and maximised over all their children's values:

1) The maximum and average ghost score $S_{\text {ghost }}$.

2) The maximum and average pill-score $S_{\text {pill }}$.

3) The maximum and average survival rate $S_{\text {survival }}$.

The values are used when determining $v_{i}$ during selection, backpropagation and deciding the move to make.

Ikehata and Ito [10] used a search tree restricted in depth to a fixed number of edges, without regard for the distance these edges represent in-game. Although the search tree in this paper is constructed similarly, the search path is variably determined by a threshold path-length $T_{\text {path }}$. A leaf is only expanded if the length of the path to the root node does not exceed $T_{\text {path }}$ (Figure 3). The variable-depth search prevents the agent from choosing 'quick fixes' when in danger, i.e. it may be safer to traverse a short path in the game when Pac-Man is in danger, than a long path which could be the case when tree-depth is limited by a fixed number of edges. Furthermore, the scoring potential over all possible paths in the tree is normalised due to the uniform length of each path.

\section{B. Tactics}

According to the current game state a tactic [10] for determining the behaviour of Pac-Man is selected. Tactics are based on the three sub-goals of Pac-Man. At any time one of the following is active:

- The Ghost score tactic is selected if: a power-pill was eaten in a previous turn, edible ghosts are in the range of Pac-Man, and the maximum survival rate is above the threshold $T_{\text {survival }}$.

- The Pill score tactic is the default tactic. It is applied when Pac-Man is safe and there are no edible ghosts in range, and the maximum survival rate is above the threshold $T_{\text {survival }}$.

- The Survival tactic is used when the maximum survival rate of the previous or current search is below the threshold, $T_{\text {survival }}$.

The $v_{i}$ value used for selection and backpropagation is based on the current tactic. It is either the maximum survival rate, $v_{i}=S_{\text {survival }}$, when the survival tactic is active, or the current score multiplied by the survival rate, $v_{i}=S_{\text {ghost }} \times S_{\text {survival }}$ or $v_{i}=S_{\text {pill }} \times S_{\text {survival }}$, for the ghost and pill tactics, respectively. The survival rate $S_{\text {survival }}$ is interpreted as a predictive indicator that the node's reward will be achieved.

The final move to be played is determined by selecting a child from the root node with the highest maximum $v_{i}$ score over all its children, based on the current tactic. If the current tactic provides no feasible reward i.e. all scores are 0 , it is replaced according to the order in the above list. This occurs when, e.g. the nearest pill or edible ghost is out of the search tree's range. If this is the case for several consecutive moves, the endgame tactic is applied (Subsection IV-H).

\section{Selection and Expansion}

During the selection step, a policy is required to explore the tree for rewarding decisions and finally converge to the most rewarding one. Upper Confidence bound applied to Trees (UCT) [19] is derived from the UCB1 function [22] for maximising the rewards of a multi-armed bandit. UCT balances the exploitation of rewarding nodes whilst allowing exploration of lesser visited nodes. The policy that determines which child to select given the current node is the one that maximises the following equation:

$$
v_{i}+C \sqrt{\frac{\ln n_{p}}{n_{i}}}
$$

$v_{i}$ is the score of the current child based on the active tactic, defined in Subsection IV-B. In the second term, $n_{p}$ is the visit count of the node and $n_{i}$ the visit count of the current child. $C$ is the exploration constant to be determined by experimentation.

UCT is applied when the visit count of a child node is above a threshold $T$. When a node's visit count is below this threshold, a child is selected randomly. In the case of Ms Pac-Man, the threshold used is 3, which ensures a higher confidence on the safety of the path. An otherwise safe and/or rewarding node may have resulted in a loss the first time it is expanded, due to the non-deterministic nature of the game. Using the threshold ensures that this node is explored again, increasing the confidence on the safety of the decision.

\section{Playout}

During the playout, Pac-Man and the ghost team make moves in a fully functional game state. Playout consists of two phases: 1) the tree phase, in which moves by Pac-Man are made according to the nodes selected during the selection phase, and 2) the playout phase, in which moves by PacMan are performed according to a randomised playout strategy described in Subsection IV-F.

During the tree phase, the path represented by the nodes selected during the selection phase is traversed. Moves corresponding to each selected node during the selection phase 
are performed by Pac-Man. Meanwhile, the ghosts move according to the playout strategy (Subsection IV-F). This provides the basis for determining the achievable score of the selected path while allowing for Pac-Man to be captured by the simulated ghost team. If Pac-Man does not lose a life during the tree phase, and the junction represented by the leaf node is reached, the playout phase starts. Both Pac-Man and the ghosts move according to the playout strategy.

In two cases, the tree phase can be interrupted due to a change in the game state unpredictable by the search tree:

1) If Pac-Man loses a life during the tree phase, the playout phase is started from the last-visited junction. Losing a life during the tree phase is basically a suicide-move, as Pac-Man may only travel forward. Therefore the playout can still be run to determine whether Pac-Man could have avoided the loss of life.

2) Pac-Man eats a ghost or a power pill, in this case the playout phase is started immediately.

A game of Ms Pac-Man ends when either Pac-Man loses all lives, or the $16^{\text {th }}$ level is cleared. It is neither useful nor computationally achievable within the strict time limit of 40 ms., to run a playout until one of these conditions holds.

The goal of the playouts is to determine the short- and longterm safety and reward of a selected path. Therefore different stopping conditions for playouts should be used. Two natural stopping conditions can be considered, either Pac-Man loses a life (dies), or the game progresses to the next maze. However, to prevent lengthy playouts, additional stopping conditions are to be introduced. Therefore during the playout phase, moves are made until one of the following four conditions applies:

1) A pre-set number of time units $T_{\text {time }}$ have passed.

2) Pac-Man is considered dead, i.e.:

- Came into contact with a non-edible ghost.

- Is trapped at the end of the playout, every available path is blocked by ghosts.

3) The next maze is reached.

4) Pac-Man eats a power pill while edible ghosts are active. As penalty, this is considered as not surviving the playout.

When the playout ends for any of the aforementioned reasons, Pac-Man's score is determined based on the three subgoals of the game. Results of a playout consist of three values:

$\bullet$

$$
R_{\text {survival }}= \begin{cases}0 & \text { if Pac-Man died } \\ 1 & \text { if Pac-Man survived }\end{cases}
$$

- $R_{\text {pill }} \in[0,1]$ the number of pills eaten, normalised by the number of pills at the root.

- $R_{\text {ghost }} \in[0,1]$ the number of ghosts eaten, normalised by the number of ghosts in the ghost team.

The goal for Pac-Man during the playout is acquiring the highest score possible whilst avoiding a loss of life. The ghosts have three goals: 1) ensure that Pac-Man loses a life by trapping her, i.e. every possible path leads to a nonedible ghost, 2) ensure the lowest ghost-reward $R_{\text {ghost }}$, which increases when Pac-Man eats edible ghosts, and 3) limit as much as possible the number of pills Pac-Man can eat.

\section{E. Long-Term Goals}

Time is an important aspect of the game. Ghosts need to be eaten as fast as possible, such that they do not return to their normal state when edible. Moreover, remaining in a maze longer than necessary increases the risk of being captured. Furthermore, after 10,000 points, Pac-Man gains a life. These are examples of long-term goals in the game. Any MCTS implementation looks at short-term rewards when running playouts. To estimate the rewards of these long-term goals, specific results are considered for both pill and ghost rewards.

To encode the long-term goal in the playouts' ghost reward $R_{\text {ghost }}$, for every eaten ghost its reward is $t_{\text {edible }}(g)$, the edible time remaining before the ghost was eaten. This ensures that ghosts eaten early are preferred over ghosts eaten later. Furthermore, when Pac-Man eats a power-pill (while no edible ghosts are active) during playout, she must achieve a ghost score higher than 0.5 at the end of the playout. If this is not the case, i.e. the ghosts were too far away to be eaten in time, the pill-reward $R_{\text {pill }}$ is 0 . If the minimum ghost score of 0.5 is achieved after eating a power-pill, the pill-reward is increased: $R_{\text {pill }}=R_{\text {pill }}+R_{\text {ghost }}$. This rapid change in scores enables PacMan to wait for the ghosts to be close enough before eating a power-pill.

The risk of being captured increases, the longer Pac-Man remains in a maze. As there are only four power pills available per maze to provide a guaranteed safe escape, eating all pills before the game naturally progresses to the next maze is a beneficial long-term goal. Therefore, points for eating pills are only added to $R_{\text {pill }}$ during playout when the current edge has been cleared, i.e. the last pill on the edge is eaten. It ensures that Pac-Man prefers to eat all pills on the edges she visits, rather than leaving isolated pills which become hard to reach as the game progresses.

\section{F. Playout Strategy}

During playout, Pac-Man and the ghost team's moves are simulated simultaneously in a fully functional game state, using the complete ruleset of the game (Section II). Both the ghosts and Pac-Man have the possibility to store moves as a Last-Good-Reply (LGR) [16]. Any time the ghost team traps Pac-Man during playout, the ghosts' moves based on Pac-Man's last visited junction are remembered. Similarly, Pac-Man's moves at junctions are remembered each time she survives a playout. Otherwise moves are forgotten [23] and no longer part of the LGR move-policy. In this subsection the playout strategies for the ghosts and Pac-Man are detailed. The strategies were designed to ensure that any time PacMan does not survive the playout (Subsection IV-D), it is due to all possible paths being blocked by ghosts. Therefore, the $S_{\text {survival }}$ score stored at nodes in the tree can be considered as an indicator of the probability of a pincer move occurring along the path [10]. 
GHOST Playout STRATEGY. The goal of the ghosts is to trap Pac-Man in such a way that every possible move leads to a path blocked by a ghost, i.e. a pincer move. The ghosts therefore are assigned a random target-location vector target that determines whether an individual ghost is to approach the front or rear of Pac-Man.

Ghosts move based on an $\varepsilon$-greedy strategy [24, 25]. With a probability $\varepsilon=0.05$ at each turn, a ghost makes a random move. With probability $1-\varepsilon$ the ghosts move according to strategic rules, derived from the rules proposed in [10]. For the ghosts there are two exclusive cases to consider, i.e. not-edible or edible, when selecting a move during playout. Moreover, there is a third case which overrules a selected move in any case.

Case 1, if ghost $g_{i}$ is not edible. A move is selected according to the following rules:

1) If the ghost can make a move that can immediately trap Pac-Man it is performed.

2) If a move, that is a Last-Good-Reply, is available based on Pac-Man's last junction, it is performed.

3) If the ghost is in the immediate vicinity of Pac-Man, i.e. within 10 distance units, the ghost moves along the next direction of the shortest path to Pac-Man.

4) If the ghost is on a junction directly connected to the edge that Pac-Man is located on, the ghost chooses the move that leads to this edge.

5) Otherwise, the ghost moves closer to the assigned target location. Based on the value of $\operatorname{targ}_{i} t_{i}$ this is either the nearest junction in front or behind Pac-Man.

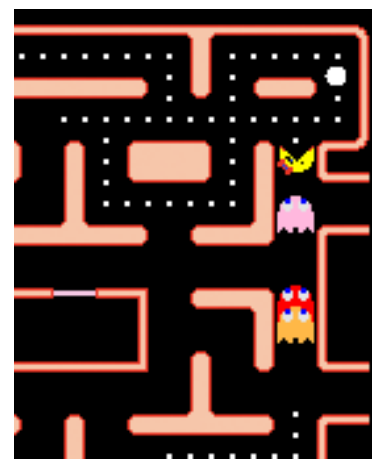

Fig. 4. Ghosts chasing Pac-Man from similar distance and location.

Case 2, if ghost $g_{i}$ is edible, a move is chosen that maximises the distance between him and Pac-Man.

Case 3, if a ghost is to move on an edge currently occupied by another ghost moving in the same direction, the move is eliminated from the ghost's selection and a different move is selected randomly. This policy ensures that ghosts are spread out through the maze, increasing their possible trapping or catching locations. It also prevents multiple ghosts from chasing Pac-Man at the same (or similar) distance and location shown in Figure 4.

PAC-MAN PLAYOUT STRATEGy. Moves made by Pac-Man are prioritised based on safety and possible reward. When more than one move has the highest priority, a random tiebreaking rule is applied. Before discussing the strategy in detail, the concept of a safe move has to be defined first. A safe move is any move that leads to an edge which:

- Has no non-edible ghost on it moving in Pac-Man's direction.

- Next junction is safe, i.e. in any case Pac-Man will reach the next junction before a non-edible ghost.

During the playout Pac-Man moves according to the following set of rules. If Pac-Man is at a junction the following rules apply, sorted by priority:

1) If a safe move that is a Last-Good-Reply is available, it is performed.

2) If a safe move leads to an edge that is not cleared, i.e. contains any number of pills, it is performed.

3) If all safe edges are cleared, select a move leading to a safe edge.

4) If no safe moves are available, a random move is selected.

If Pac-Man is on an edge, she can either choose to maintain her current path or reverse course. The following rules consider the cases when Pac-Man is allowed to reverse:

- There is a non-edible ghost heading for Pac-Man on the current path.

- A power-pill was eaten, in this case the move which leads to the closest edible ghost is selected.

- A ghost in the edible state was eaten, the move which leads to the next closest edible ghost is selected.

In any other case Pac-Man continues forward along the current edge. Note that, if Pac-Man previously chose to reverse on the current edge, she may not reverse again until she reaches a junction.

\section{G. Backpropagation}

Results are back-propagated from the expanded leaf node to the root based on maximum backpropagation [18]. Scores stored at each node represent the maximum scores of its children based on $v_{i}$ according to the current tactic (Subsection IV-B). Whereas most games use average backpropagation, maximisation is chosen since each move at a junction can have altogether different results [10]. For example, at a junction Pac-Man has two options to move. A decision to go left may lead to a loss of life for Pac-Man in all playouts, whereas a choice to go down is a determined to be safe in every playout. When using averaged values, the resulting survival rate is 0.5 , whereas maximum backpropagation results in the true survival rate of 1 .

\section{H. Endgame Tactics}

During the final moments in a maze, or when Pac-Man is located in an isolated area of the maze, the nearest possible reward, i.e. an edible ghost or a pill, may be out of the search tree's range (Figure 5). These cases are considered as the endgame, Pac-Man will no longer be motivated to choose one move over another due to the lack of rewards. This leads 


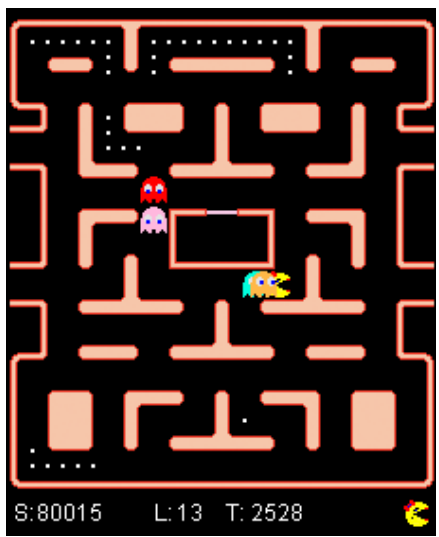

Fig. 5. Example of an endgame situation, maze_time $>2000$ and the nearest pill is outside the search tree's range.

to a continuous fall back to the survival tactic as defined in Subsection IV-B. This is problematic because the survival tactic only provides motivation when Pac-Man is in danger of being eaten by the ghosts. In this case, the endgame tactic is applied when one of the following criteria holds:

1) No move could be selected based on the active tactic for 5 consecutive moves, i.e. $S_{\text {pill }}=0$ or $S_{\text {ghost }}=0$.

2) The maze_time $>2000$, i.e. the time Ms. Pac-Man was in the current maze is higher than 2000 time units.

When the endgame tactic is active, similar to [7] and [9] a target location is selected based on a heuristic evaluation of the game state. The pseudo-code for the algorithm used to select the current target $t$ is listed in Algorithm 1. A new target is selected each time Pac-Man is at a junction.

When a target is set, at the end of the playout phase $R_{\text {pill }}$ (Subsection IV-D) is replaced by:

$$
R_{\text {target }}= \begin{cases}0 & \Delta \text { Dist } \leq 0 \\ \Delta D i s t & \Delta \text { Dist }>0 \\ 1 & \text { Target location was reached }\end{cases}
$$

where $\Delta D i s t$ is the normalised difference in distance to $t$ at the start and end of the playout.

If the endgame tactic was only applied for the first reason, thus maze_time $<2000$, it is possible to terminate the endgame tactic, returning to one of the default tactics discussed in Subsection IV-B. This occurs when a move is selected with a score, $S_{\text {pill }}$ or $S_{\text {ghost }}$ based on the active tactic, of at least 0.5 , implying that there is again sufficient motivation to select a move based on one of the default tactics.

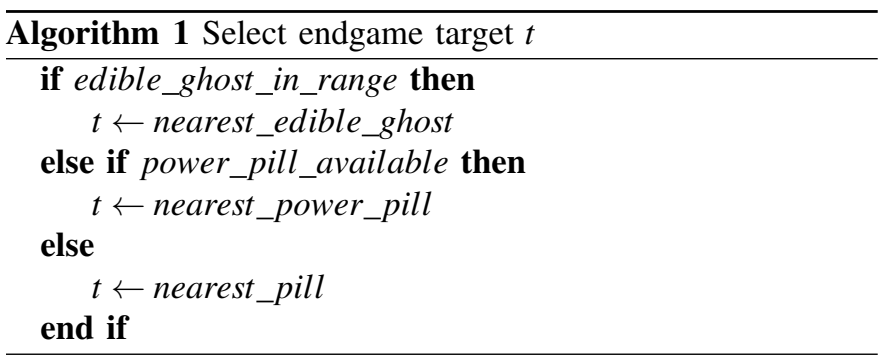

\section{EXPERIMENTS}

In the following subsections the experimental setup will be detailed, and experimental results discussed.

\section{A. Experimental setup}

The MCTS PAC-MAN agent was implemented using the framework provided by the Ms Pac-Man vs Ghosts competition [2]. Furthermore, an MCTS GHOST TEAM using enhancements within the MCTS framework discussed in this paper was developed. The MCTS GHOST TEAM uses the strategic playout and tactics discussed in this paper. However, due to the difference in goals and implementation between Pac-Man and the ghost team, the MCTS GHOST TEAM uses a constant depth tree of 5 nodes, no endgame tactics, and adjusted longterm goals.

The version of the Ms Pac-Man game framework used is WCCI12_1.1. It includes pre-computed distances for the four mazes, providing a fast method for determining shortest paths and distances. Both the framework and the agent were developed in Java.

Results are comprised of the average, maximum and minimum score, the average number of lives remaining and average maze reached at the terminal state of the game. Average scores are rounded to the nearest integer. Each time 100 runs are performed, allowing the agents the official $40 \mathrm{~ms}$. to run playouts and compute a move.

The following values, determined by trial-and-error, were used for the parameters discussed in the paper: the minimum survival rate $T_{\text {survival }}=0.7$, the maximum variable tree depth $T_{\text {path }}=55$, the maximum time units per playout phase $T_{\text {time }}=$ 80 , and a UCT constant $C=1.5$ was used.

To determine the influence of the proposed enhancements, results are compared to agents with a single enhancement disabled. Additional experiments are run using agents with, 1) a fixed node depth-limit, 2) no endgame tactic, 3) a randomised playout strategy, and 4) the Last-Good-Reply policy disabled.

\section{B. Results}

Experiments were run to evaluate the performance of the MCTS PAC-MAN agent against the benchmarking ghost team LEGACY2THERECKINING (LEGACY2) provided by the competition framework. Furthermore, the agent's performance is tested against the MCTS GHOST TEAM developed using the framework discussed in this paper.

Table I shows the resulting scores of our MCTS PACMAN agent versus the benchmarking team LEGACY2 and the MCTS GHost TEAM. For comparison the same ghost teams played 100 games against the STARTER PAC-MAN agent which uses a limited rule set. From this we can conclude that the MCTS GHOST TEAM outperforms LEGACY2 when playing against both the MCTS PAC-MAN and STARTER PAC-MAN agents. Moreover, it is clear that the MCTS PACMAN agent outperforms the STARTER PAC-MAN by far.

Currently, no official competition results in which the WCCI12_1.1 version of the Ms Pac-Man framework was used exist. Past competitions used a similar framework, including 
TABLE I

ACHIEVED SCORES, 100 GAMES

\begin{tabular}{|c|c|c|c|c|}
\hline \multicolumn{5}{|c|}{ Pac-Man agent: MCTS PAC-MAN } \\
\hline $\begin{array}{c}\text { Ghost Team } \\
\text { agent }\end{array}$ & $\begin{array}{c}\text { Avg. } \\
\text { score }\end{array}$ & $\begin{array}{c}\text { Max. } \\
\text { score }\end{array}$ & $\begin{array}{c}\text { Min. } \\
\text { score }\end{array}$ & $\begin{array}{c}95 \% \\
\text { conf. int. }\end{array}$ \\
\hline Legacy2 & 107,561 & 127,945 & 40,495 & 2,791 \\
\hline MCTS GHost TEAm & 36,477 & 65,195 & 2,830 & 2,498 \\
\hline \multicolumn{5}{|c|}{ Pac-Man agent: STARTER PAC-MAN } \\
\hline $\begin{array}{c}\text { Ghost Team } \\
\text { agent }\end{array}$ & $\begin{array}{c}\text { Avg. } \\
\text { score }\end{array}$ & $\begin{array}{c}\text { Max. } \\
\text { score }\end{array}$ & $\begin{array}{c}\text { Min. } \\
\text { score }\end{array}$ & $\begin{array}{c}95 \% \\
\text { conf. int. }\end{array}$ \\
\hline LeGaCy2 & 4,260 & 9,050 & 1,460 & 280 \\
\hline MCTS GHoSt Team & 2,799 & 5,980 & 1,040 & 211 \\
\hline
\end{tabular}

the benchmarking ghost team LEGACY2. However, it is not the case that the underlying data structures provided in the current version of the software provide an unfair advantage to either the ghost team or Pac-Man. Moreover, since the LEGACY2 ghost team's rule-base has remained the same, a rough comparison may be drawn. In Table II, the top-3 scoring Pac-Man controllers during the CIG'11 [26] are presented with their achieved scores versus the LEGACY2 ghost team. Because the results of these agents differ substantially from our MCTS PAC-MAN agent it is safe to conclude that the performance has increased. An average performance gain of 40,962 points, based on the top scoring Pac-Man agent during the CIG' 11 , is achieved by our MCTS agent.

TABLE II

CIG'11 RANKINGS, 10 GAMES

\begin{tabular}{|c|c|c|c|c|c|}
\hline \multicolumn{7}{|c|}{ Ghost Team: LegACY2 } \\
\hline & $\begin{array}{c}\text { Pac-Man } \\
\text { agent }\end{array}$ & $\begin{array}{c}\text { Avg. } \\
\text { score }\end{array}$ & $\begin{array}{c}\text { Max. } \\
\text { score }\end{array}$ & $\begin{array}{c}\text { Min. } \\
\text { score }\end{array}$ & $\begin{array}{c}95 \% \\
\text { conf. int. }\end{array}$ \\
\hline 1 & Spooks & 66,599 & 76,080 & 35,270 & 7,378 \\
\hline 2 & PhanToMMENACE & 56,313 & 88,090 & 30,350 & 13,311 \\
\hline 3 & ICEPAMBuSH_CIG11 & 20,619 & 29,320 & 9,160 & 4,384 \\
\hline \hline- & MCTS Pac-Man & 107,561 & 127,945 & 40,495 & 2,791 \\
\hline
\end{tabular}

To test the proposed enhancements for the MCTS agent, 100 games per enhancement were played against the LEGACY2 ghost team. The enhancements were individually disabled or defaulted by the following:

- The playout strategy was replaced by a random strategy for both the ghosts and Pac-Man. That is, Pac-Man cannot reverse and chooses each move randomly, and the ghosts choose the path that leads closer to Pac-Man.

- A constant tree-depth of 4 ply, determined by trial-anderror, replaces the variable depth tree enhancement.

- The Last-Good-Reply (LGR) policy was disabled.

- The endgame tactic was disabled altogether. Only the default strategies can be used when selecting a move.

Results of these games are shown in Table III. Note that the Long-Term goals were not disabled in any test, as there is no immediate default policy for scoring playouts.

The random playout has the largest impact on overall scoring, since MCTS is dependent on the results of its playouts to determine the best move. It is followed by the constant tree-depth, which causes discrepancies when determining the scoring potential and survival rate over each path in the tree.

Both the LGR policy and endgame tactic have a low impact on the results, lives remaining, and maze reached. However, it is likely that against more advanced ghost teams these enhancements play a more important role. Because the LEGACY2 ghost team was not designed to trap Pac-Man, the increase in performance may be lower than were the agent to perform against more intelligent ghost teams.

According to Table IV, for both the disabled random playouts and constant tree depth, the number of remaining lives at the end of each run, and the average maze reached is lower. Implying increased survivability of the agent due to these enhancements.

TABLE III

DiSABLED ENHANCEMENTS, SCORES, 100 GAMES

\begin{tabular}{|c|c|c|c|c|}
\hline \multicolumn{5}{|c|}{ Ghost Team: LEGACY2, Pac-Man agent: MCTS PAC-MAN } \\
\hline $\begin{array}{c}\text { Enhancement } \\
\text { disabled }\end{array}$ & $\begin{array}{c}\text { Avg. } \\
\text { score }\end{array}$ & $\begin{array}{c}\text { Max. } \\
\text { score }\end{array}$ & $\begin{array}{c}\text { Min. } \\
\text { score }\end{array}$ & $\begin{array}{c}95 \% \\
\text { conf. int. }\end{array}$ \\
\hline Strategic playout & 44,758 & 65,270 & 11,900 & 2,310 \\
\hline Var. depth tree & 101,836 & 124,925 & 43,595 & 3,326 \\
\hline Last-Good-Reply & 105,723 & 125,885 & 45,830 & 2,964 \\
\hline Endgame tactic & 108,020 & 125,440 & 40,945 & 2,551 \\
\hline \hline MCTS PAC-MAN & 107,561 & 127,945 & 40,495 & 2,791 \\
\hline
\end{tabular}

TABLE IV

DisABLED ENHANCEMENTS, STATISTICS, 100 GAMES

\begin{tabular}{|c|c|c|c|c|}
\hline \multicolumn{5}{|c|}{ Ghost Team: LEGACY2, Pac-Man agent: MCTS PAC-MAN } \\
\hline $\begin{array}{c}\text { Enhancement } \\
\text { disabled }\end{array}$ & $\begin{array}{c}\text { Avg. lives } \\
\text { remaining }\end{array}$ & $\begin{array}{c}95 \% \\
\text { conf. int. }\end{array}$ & $\begin{array}{c}\text { Avg. level } \\
\text { reached }\end{array}$ & $\begin{array}{c}95 \% \\
\text { conf. int. }\end{array}$ \\
\hline Strategic playout & 0.55 & 0.19 & 12.76 & 0.70 \\
\hline Var. depth tree & 1.21 & 0.24 & 14.32 & 0.53 \\
\hline Last-Good-Reply & 1.78 & 0.25 & 15.01 & 0.45 \\
\hline Endgame tactic & 1.70 & 0.24 & 15.21 & 0.39 \\
\hline \hline MCTS PAC-MAN & 1.76 & 0.25 & 15.19 & 0.41 \\
\hline
\end{tabular}

\section{Conclusion \& Future Research}

The discussed enhancements for the Monte-Carlo Tree Search (MCTS) framework have resulted in a Pac-Man agent achieving a high score of 127,945 points versus the LEGACY2THERECKONING ghost team. Regarding the results of previous competitions, an average performance gain of 40,962 points, based on the top scoring Pac-Man agent during the CIG'11, is achieved by our MCTS agent. Additional experiments reveal that the variable depth tree and strategic playout ensure the highest increase in performance. Although the endgame tactics and Last-Good-Reply policy did not increase the final scores significantly, they may be crucial to competing with more advanced ghost teams. However, it is possible that when the playout strategy is further improved, LGR will have less effect on overall scores. Based on the results we may conclude that the MCTS framework makes strong Pac-Man agents possible.

The performance of the MCTS agent could be improved by using Heat-maps [10] to determine the most dangerous 
locations in a maze to enhance the pill-reward. Furthermore, although the proposed ghost playout strategy is designed to maximise the possibility of a pincer-move, ghosts do not always capture Pac-Man when possible in playouts. To improve the playout-phase further two improvements could be made. 1) N-Grams [27], when applied to the playout phase may improve the possibility of Pac-Man being caught whenever possible, increasing the confidence of the safety of moves in the search tree. 2) The knowledge rules of fast rule-based agents from the upcoming competitions could be used if they perform well.

\section{REFERENCES}

[1] "Ms Pac-Man Competition, screen-capture," http:// dces.essex.ac.uk/staff/sml/pacman/PacManContest.html, accessed May, 2012.

[2] P. Rohlfshagen and S. M. Lucas, "Ms Pac-Man Versus Ghost Team CEC 2011 competition," Proc. of the IEEE Congress on Evolutionary Computation, pp. 7077, 2011.

[3] A. M. Alhejali and S. M. Lucas, "Evolving diverse Ms. Pac-Man playing agents using genetic programming," in Workshop on Comput. Intell. (UKCI). IEEE, 2010, pp. $1-6$.

[4] S. M. Lucas, "Evolving a neural network location evaluator to play Ms. Pac-Man," in Proc. of the IEEE Comput. Intell. and Games. IEEE, 2005, pp. 203-210.

[5] D. Robles and S. M. Lucas, "A simple tree search method for playing Ms. Pac-Man," in IEEE Comput. Intell. and Games. IEEE, 2009, pp. 249-255.

[6] C. Browne, E. Powley, D. Whitehouse, S. M. Lucas, P. I. Cowling, P. Rohlfshagen, S. Tavener, D. Perez, S. Samothrakis, and S. Colton, "A survey of Monte-Carlo tree search methods," IEEE Trans. Comput. Intell. AI in Games, vol. 4, no. 1, pp. 1-43, 2012.

[7] S. Samothrakis, D. Robles, and S. M. Lucas, "Fast approximate max-n Monte-Carlo tree search for ms pacman," IEEE Trans. Comput. Intell. AI in Games, vol. 3, no. 2, pp. 142-154, 2011.

[8] B. K. B. Tong and C. W. Sung, "A Monte-Carlo approach for ghost avoidance in the Ms. Pac-Man game," in Int. IEEE ICE-GIC. IEEE, 2011, pp. 1-8.

[9] B. K. B. Tong, C. M. Ma, and C. W. Sung, "A MonteCarlo approach for the endgame of Ms. Pac-Man," in IEEE Comput. Intell. and Games (CIG). IEEE, 2011, pp. 9-15.

[10] N. Ikehata and T. Ito, "Monte-Carlo tree search in Ms. Pac-Man," in IEEE Comput. Intell. and Games (CIG). IEEE, 2011, pp. 39-46.

[11] R. Thawonmas and T. Ashida, "Evolution strategy for optimizing parameters in Ms Pac-Man controller ICE Pambush 3," in IEEE Comput. Intell. and Games (CIG), 2010, pp. 235-240.

[12] "ICE gUCT (nqkien) simulation based ghost team controller report," http://cec11.pacman-vs-ghosts.net/ viewReport.php?id=10, accessed, july 2012.
[13] "ICEgUCT_CIG11 simulation based ghost team controller report," http://cig11.pacman-vs-ghosts.net/report. php?id=44, accessed, july 2012.

[14] "ICEpAmbush_CIG11 simulation based Ms Pac-Man controller report," http://cig11.pacman-vs-ghosts.net/ report.php?id=50, accessed, july 2012.

[15] "CEC11 Ms Pac-Man vs Ghosts Competition rankings," http://cec11.pacman-vs-ghosts.net/rankings.php, accessed, july 2012.

[16] P. D. Drake, "The last-good-reply policy for Monte-Carlo Go," ICGA Journal, vol. 32, no. 4, pp. 221-227, 2009.

[17] G. M. J.-B. Chaslot, M. H. M. Winands, H. J. van den Herik, J. W. H. M. Uiterwijk, and B. Bouzy, "Progressive strategies for Monte-Carlo tree search," New Mathematics and Natural Computation, vol. 4, no. 3, pp. 343-357, 2008.

[18] R. Coulom, "Efficient selectivity and backup operators in Monte-Carlo tree search," in Computers and games: 5th Int. conference (CG), vol. 4630. Springer, 2007, pp. 72-83.

[19] L. Kocsis and C. Szepesvári, "Bandit based MonteCarlo planning," in Machine Learning: ECML 2006, ser. LNCS. Springer, 2006, vol. 4212, pp. 282-293.

[20] A. Rimmel, O. Teytaud, C.-S. Lee, S.-J. Yen, M.-H. Wang, and S.-R. Tsai, "Current frontiers in computer Go," IEEE Trans. Comput. Intell. AI in Games, vol. 2, no. 4, pp. 229-238, 2010.

[21] B. Arneson, R. B. Hayward, and P. Henderson, "MonteCarlo tree search in Hex," IEEE Trans. Comput. Intell. AI in Games, vol. 2, no. 4, pp. 251-258, 2010.

[22] P. Auer, N. Cesa-Bianchi, and P. Fischer, "Finite-time analysis of the multiarmed bandit problem," Machine learning, vol. 47, no. 2-3, pp. 235-256, 2002.

[23] H. Baier and P. D. Drake, "The power of forgetting: Improving the last-good-reply policy in Monte Carlo Go," IEEE Trans. Comput. Intell. AI in Games, vol. 2, no. 4, pp. 303-309, 2010.

[24] R. S. Sutton and A. G. Barto, Reinforcement learning: An introduction. MIT Press, 1998.

[25] N. Sturtevant, "An analysis of uct in multi-player games," in Computers and Games, ser. LNCS. Springer, 2008, vol. 5131, pp. 37-49.

[26] "Ms Pac-Man vs Ghost competition CIG11 ranknings," http://cig11.pacman-vs-ghosts.net/rankings.php, accessed, May 2012.

[27] M. J. W. Tak, M. H. M. Winands, and Y. Björnsson, "Ngrams and the last-good-reply policy applied in general game playing," IEEE Trans. Comput. Intell. AI in Games, vol. 4, no. 2, pp. 73-83, 2012. 